Physics

Physics Research Publications

X-ray flaring activity of Markarian 421

W. Cui 
The Astrophysical Journal, 605:662-669, 2004 April 20

(C) 2004. The American Astronomical Society. All rights reserved. Printed in U.S.A.

\title{
X-RAY FLARING ACTIVITY OF MARKARIAN 421
}

\author{
WEI CUI \\ Department of Physics, Purdue University, West Lafayette, IN 47907; cui@physics.purdue.edu \\ Received 2003 November 26; accepted 2004 January 7
}

\begin{abstract}
We report results from a systematic search for X-ray flares from Mrk 421, using archival data from the Rossi $X$-Ray Timing Explorer ( $R X T E)$ satellite. The flares are clearly seen over a wide range of timescales. The quasicontinuous coverage of the source with the All-Sky Monitor (ASM) reveals frequent occurrence of major flares that last for months. On a few occasions, the source was intensively monitored with the more sensitive pointing instruments aboard $R X T E$. The data from these observations show the presence of X-ray flares of much shorter duration, ranging from weeks down to less than an hour. For the first time, we clearly resolved the most rapid flares from Mrk 421. Moreover, Fourier analyses reveal variability on even shorter timescales, up to about $0.01 \mathrm{~Hz}$. The source appears to behave differently in its spectral properties during different flares, large or small, which is intriguing. While significant hysteresis is observed to be associated with spectral evolution in some cases, little is seen in other cases. Sometimes, the shape of the X-ray spectrum hardly varies across a flare. Therefore, the phenomenology is complex. The observed hierarchical structure of the X-ray flares seems to imply the scaleinvariant nature of the phenomenon, perhaps similar in this regard to solar flares or the rapid X-ray flares observed from stellar-mass black holes. Combined with other results, the observed flaring timescales seriously constrain the physical properties of X-ray-emitting regions in the jets of Mrk 421.
\end{abstract}

Subject headings: BL Lacertae objects: individual (Markarian 421) — galaxies: active radiation mechanisms: nonthermal - X-rays: galaxies

\section{INTRODUCTION}

Mrk 421 is one of the brightest extragalactic X-ray sources in the sky. It belongs to the group of BL Lac objects, a subclass of radio-loud active galactic nuclei (AGNs). The BL Lac objects are known to be highly variable at nearly all wavelengths and their spectral energy distributions (SEDs) are thought to be dominated by radiation from relativistic particles in the jets that propagate roughly along the line of sight (Urry \& Padovani 1995). Mrk 421 is also one of the few BL Lac objects that were discovered to emit strongly at $\mathrm{TeV}$ energies in the past decade (Punch et al. 1992). In fact, the total energy output of Mrk 421 in gamma-rays seems comparable to that in X-rays (Buckley et al. 1996; Maraschi et al. 1999; Krawczynski et al. 2001). The spectral energy distribution (SED) of the source is dominated by the X-ray and gamma-ray emission. While the origin of gamma-ray photons is still being debated, it is generally agreed that X-ray photons originate mostly in the synchrotron radiation from highly relativistic electrons in the jets.

BL Lac objects are known for their flaring activities. X-ray flares with duration of longer than a day have been seen from Mrk 421 (Tanihata et al. 2001; Fossati et al. 2000; Brinkmann et al. 2003). Remarkably, a strong flare was detected from the source at $\mathrm{TeV}$ energies that lasted only for about an hour (Gaidos et al. 1996). The TeV flare showed significant substructures that were of even shorter durations. The origin of $\mathrm{X}$-ray or TeV flares is hardly understood. The flares are often thought to be related to internal shocks in the jets of a blazar (Rees 1978; Spada et al. 2001), or to major ejection events of new components of relativistic plasma into the jet (e.g., Böttcher et al. 1997; Mastichiadis \& Kirk 1997). Despite such uncertainty, the measured short flaring timescale alone has already severely constrained the size of the $\mathrm{TeV}$ emission region and the Doppler factor ${ }^{1}$ of the jets (Gaidos et al. 1996), as well as the accretion process in the system (Celotti, Fabian, \& Rees 1998). If X-ray and TeV photons are associated with the same population of emitting electrons, as is often postulated based on the observed correlated behaviors of the source in the two energy bands (e.g., Maraschi et al. 1999), then X-ray flares of similarly short duration should also occur. In this context, we conducted a systematic search for such flares, making use of the rich database on Mrk 421 provided by the Rossi X-Ray Timing Explorer (RXTE).

\section{DATA}

Mrk 421 is one of the X-ray sources that has been frequently observed with $R X T E$ over the past $8+$ years. It is certainly the most observed blazar. The $R X T E$ observations often represent X-ray coverage of Mrk 421 in a multi-wavelength campaign. Archival data are now available from campaigns conducted in 1996 (under the Guest Observing programs 10341 and 10345), 1997 (20341), 1998 (30261, 30262, and 30269), 2000 (40182), and 2001 (50190 and 60145). The great majority of the observations were made in "snapshot" modes, with effective exposure times ranging from half a kilosecond to several kiloseconds. Deeper observations (10-20 ks) were occasionally carried out. In the following, we briefly describe the $R X T E$ instruments and the data reduction procedures adopted for this work.

\subsection{Instruments}

$R X T E$ carries on board two co-aligned, large-area detectors (Bradt, Rothschild, \& Swank 1993), the Proportional Counter

\footnotetext{
${ }^{1}$ Defined as $\delta \equiv \Gamma^{-1}(1-\beta \cos \theta)^{-1}$, where $\Gamma$ and $\beta$ are the Lorentz factor and speed (in units of the speed of light $c$ ) of the bulk motion, and $\theta$ is the angle to the line of sight.
} 
Array (PCA) and the High-Energy X-ray Timing Experiment (HEXTE), in addition to the All-Sky Monitor (ASM). Both of the pointing instruments have a $1^{\circ}$ field of view. The PCA consists of five nearly identical proportional counter units (PCUs). It has a total collecting area of about $6500 \mathrm{~cm}^{2}$ and covers a nominal energy range of $2-60 \mathrm{keV}$. However, the operational constraints often require that some of the PCUs be turned off. Exactly which PCUs are off varies from observation to observation, but PCU 0 and PCU 2 are nearly always in operation. Further complication (to data analysis) developed following the loss of the front veto layer in PCU 0 . The result is that the data from PCU 0 are more prone to contamination by events caused by low-energy electrons entering the detector. This is particularly relevant to the study of variability of weak sources like Mrk 421.

Although the HEXTE covers a very interesting energy range (nominally $15-250 \mathrm{keV}$ ) for AGN studies, it is not very sensitive. We did analyze all of the HEXTE data for this work, but did not find it very useful, since we are mostly interested in detecting rapid X-ray flares from Mrk 421. The HEXTE results, along with other spectral results, will be presented in a future paper on broadband SED variability. We do not discuss them further here.

The ASM consists of three proportional counters, each of which has a $6^{\circ} \times 90^{\circ}$ field of view, and covers about $80 \%$ of the sky upon the completion of one full rotation, which takes about $1.5 \mathrm{hr}$. However, the orientation of the rotation axis of the ASM is fixed by the pointing configuration of the PCA and HEXTE, so there is generally no flexibility in controlling which $80 \%$ of the sky is covered at a given time. This is a major source of data gaps in the quasi-continuous light curve of a particular source, in addition to Earth occultation and proximity of the source to the Sun. The ASM scans the sky in $6^{\circ}$ steps, which are often referred to as ASM dwells. The exposure time of each dwell is $90 \mathrm{~s}$.

\subsection{Data Reduction}

Multiple data modes are usually employed in a PCA observation. Two of the available data modes are always run and are thus referred to as the standard modes Standard1 and Standard2. We rely almost exclusively on the Standard2 data for this work. The data have a time resolution of $16 \mathrm{~s}$, which is sufficient for our purposes here and covers the entire PCA passing band with 128 energy channels.

The PCA data were reduced with the latest version of FTOOLS (ver. 5.2) that is distributed as part of the software suite HEAsoft (ver. 5.2). ${ }^{2}$ For an observation, we first filtered data by following the standard procedure for faint sources (see the online RXTE Cook Book), ${ }^{3}$ which resulted in a list of good time intervals (GTIs). We then simulated background events for the observation using FTOOLS and the latest background model that is appropriate for faint sources (file: pca_bkgd_cmfaintl7_eMv20020201.mdl). Using the GTIs, we proceeded to extract a light curve from the data (combining all active PCUs) in each of the following energy bands: 2.0-5.7, $5.7-11,11-60$, and $2.0-60 \mathrm{keV}$. Note that the boundaries of each band are matched up as closely as possible across different PCA epochs but are only approximate (up to $\pm 0.2 \mathrm{keV}$ ). We repeated the steps to construct the corresponding background light curves from the simulated events. Finally, we subtracted off the background to obtain the light curves of the

\footnotetext{
${ }^{2}$ See http://heasarc.gsfc.nasa.gov/docs/software/lheasoft.

3 See http://heasarc.gsfc.nasa.gov/docs/xte/recipes/cook book.html.
}

source. In an attempt to avoid possible artifacts caused by background variations (see $\S 2.1$ ), we excluded data from PCU 0 when we made light curves from observations conducted in epoch 5 .

The ASM data are publicly available. The light curves come in three energy bands: $1.5-3,3-5$, and 5-12 $\mathrm{keV}$, providing crude spectral information about a source. We obtained the light curves of Mrk 421 from the MIT archive. ${ }^{4}$ We chose to filter out data points with error bars (on the count rates in the summed band) greater than 2.0 counts $\mathrm{s}^{-1}$, which constitutes about $10 \%$ of the data. We then weighted the raw data by $1 / \sigma^{2}$ and rebinned it to produce weekly-averaged light curves.

\section{RESULTS}

To provide a global view of flaring activity of Mrk 421, Figure 1 shows the ASM light curve of Mrk 421 for the 1.5$12 \mathrm{keV}$ band over roughly an $8 \mathrm{yr}$ period. The light curve shows two things very clearly. First, the source is highly variable in X-ray emission. It goes from below the detection threshold to a flux level of 50-60 morab in the ASM passing band. Second, frequent flares are easily identifiable from the light curve, with durations ranging from less than a month up to almost a year. There appears to have been four major flares over $8 \mathrm{yr}$, or one every $2 \mathrm{yr}$ on average, although the source seems to be more active in recent years. There is hardly any time period that resembles a "quiescent state." During the periods between the major flares, which we refer as the "low state" for convenience, the average flux of Mrk 421 is about 5 mcrab (see Fig. 1). We stress, however, that smaller (both in amplitude and duration) flares are nearly always present.

To examine possible spectral evolution of Mrk 421 during the major flares, we computed the ratio between the ASM rates in the $3-12 \mathrm{keV}$ band to those in the $1.5-3 \mathrm{keV}$ band and used it as a measure of the spectral shape. Figure 2 shows the time series of the hardness ratio that covers the periods of the two largest flares. The data are quite noisy, even with weekly

${ }^{4}$ See http://xte.mit.edu/asmlc/srcs/mkn421.html\#data.

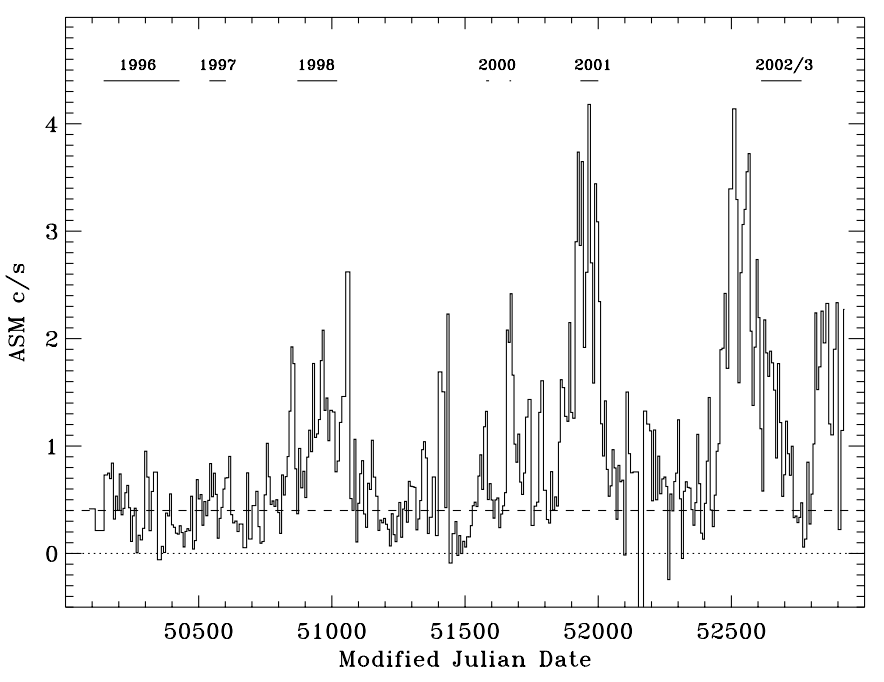

FIG. 1.-ASM light curve of Mrk 421. Each data point represents a weighted average of raw count rates over 7 consecutive days (following the exclusion of "bad data"; see text). A few negative data points still remain, which are obviously artifacts. For clarity, the error bars are not shown. The dashed line shows roughly the average count rate of the source in the low state. The periods of the monitoring campaign on Mrk 421 with the pointing instruments aboard RXTE are indicated with horizontal lines at the top of the graph. 


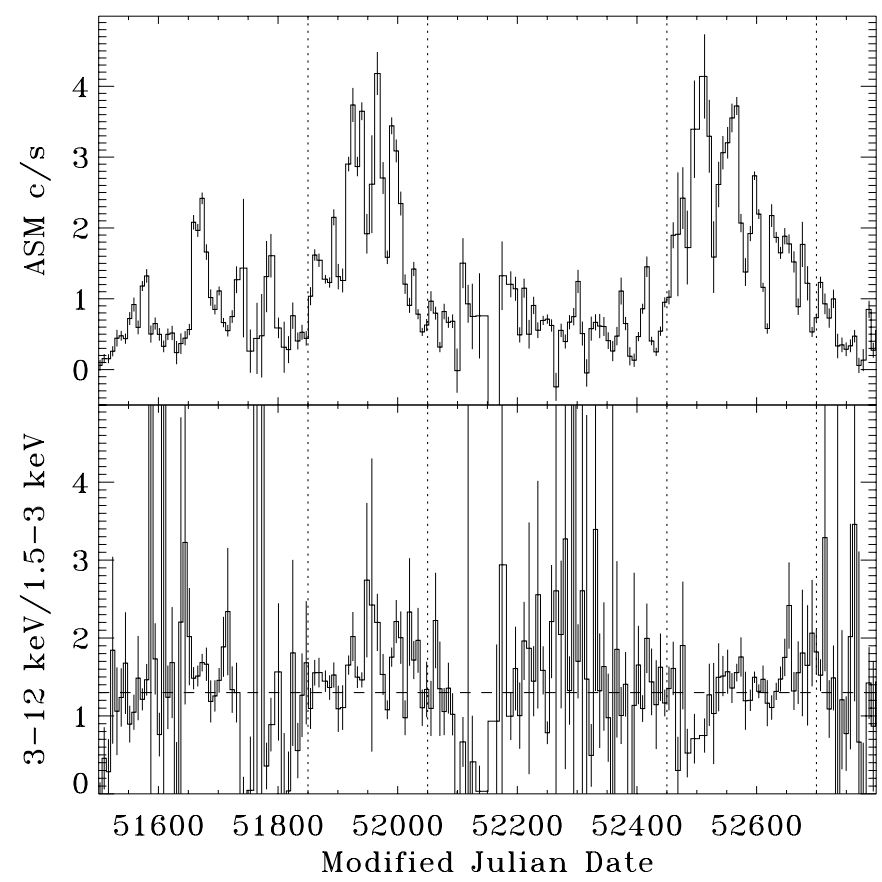

FIG. 2.-Spectral variation across major X-ray flares. Two of the flares in Fig. 1 are highlighted here. The time series of the hardness ratio in the bottom panel was derived from the weekly averaged light curves in the two energy bands. The dashed line shows roughly the nominal value for the low state.

binning, but there does not seem to be any strong spectral evolution across either flare.

\subsection{Low State}

Mrk 421 was in an extended low state throughout 1996 and 1997 (see Fig. 1). It was monitored regularly by the PCA, with particularly good coverage in 1996, and the data allow us to examine, in a more detailed manner, the properties of the source in this state. The source is by no means quiet during this period, as shown in Figure 3. With much improved sensitivity of the PCA (compared to the ASM), we now see the presence of flares of shorter durations. Two larger flares are recognizable from the figure (despite the presence of data gaps) that last for weeks. Superimposed on them are many "spikes" whose duration can be as short as about a day. The

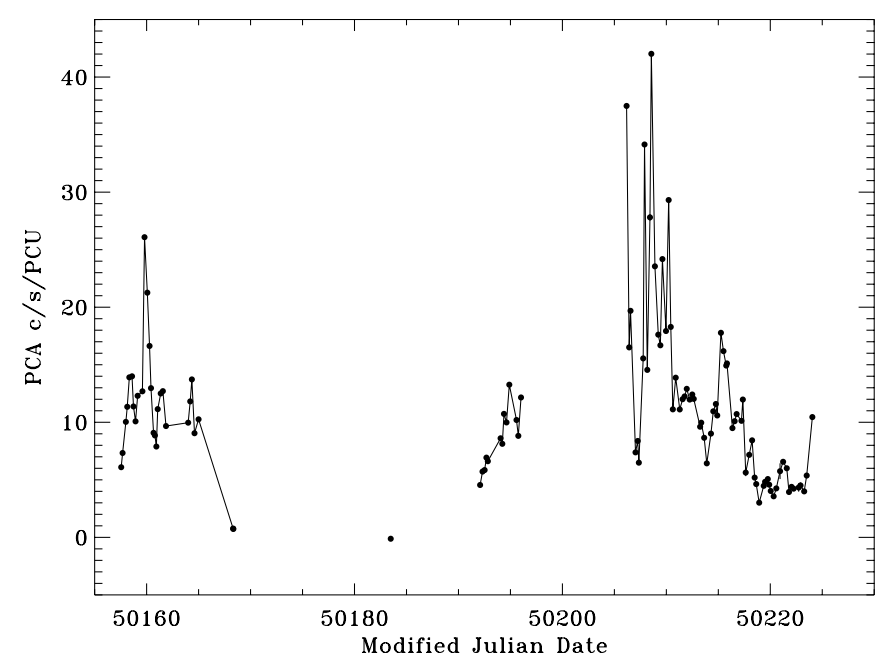

FIG. 3.-PCA light curve of Mrk 421 in the low state. Only results from the 1996 campaign are shown here. The count rates were computed for the $2-60 \mathrm{keV}$ band and were averaged with $2048 \mathrm{~s}$ time bins. source reaches the peak flux of roughly $10^{-9} \mathrm{ergs} \mathrm{cm}^{-2} \mathrm{~s}^{-1}$ (in the $2-60 \mathrm{keV}$ band) in this state but can drop below even the PCA detection threshold $\left(\lesssim 10^{-11} \mathrm{ergs} \mathrm{cm}^{-2} \mathrm{~s}^{-1}\right)$.

As with the ASM data, we defined a hardness ratio (5.7$60 \mathrm{keV} / 2-5.7 \mathrm{keV}$ ) to examine the spectral behavior of Mrk 421 during these short flares. Although crude, the hardness ratio does provide a model-independent way of presenting the data. In Figure 4, we plotted the hardness ratio against the overall count rate of the source for the two flares separately. While there seems to be a common trend of spectral hardening as the source brightens, there are also significant differences between the two cases. Significant hysteresis appears to be associated with the spectral evolution during the first flare, but little hysteresis is seen during the second one. Similar hysteresis patterns have been seen before (e.g., Takahashi et al. 1996; Kataoka et al. 2000; Zhang 2002; Giebels et al. 2002; Falcone, Cui, \& Finley 2004) and seem to be quite common among blazars. The patterns that we have observed here are complex in shape, e.g., with embedded subloops, perhaps reflecting the coexistence of flares on different timescales. Also, both clockwise- and counterclockwise-running subloops seem to be present, so the phenomenology is not simple.

We searched for flares of even shorter duration (less than a day). The effort was complicated by the presence of data gaps (mostly due to earth occultations or South Atlantic Anomaly crossings) for relatively long observations. We failed to detect any significant flares on timescales of tens of minutes to hours, although the light curve seems to show variation on these timescales in several cases. At the level of the systematic variations seen, however, one must be worried about imperfect background modeling. To illustrate the point, we show a possible flare in Figure 5, along with the corresponding light curve of the background. The variation patterns seem to be quite similar in the two cases, which is of concern. In this example, however, the observed amplitude of the variation is perhaps too large to be attributed entirely to the inaccuracy of the background model.

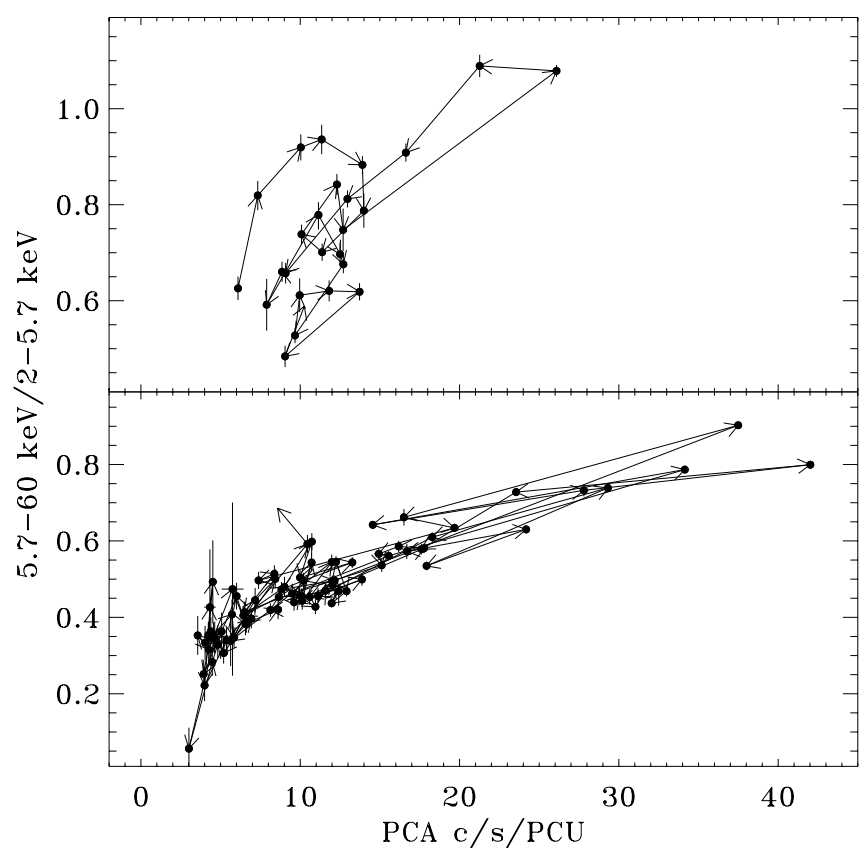

FIG. 4.- Spectral variation across short X-ray flares in the low state. The results are shown separately for the two flares shown in Fig. 3. The arrows indicate the time sequence across a flare. 


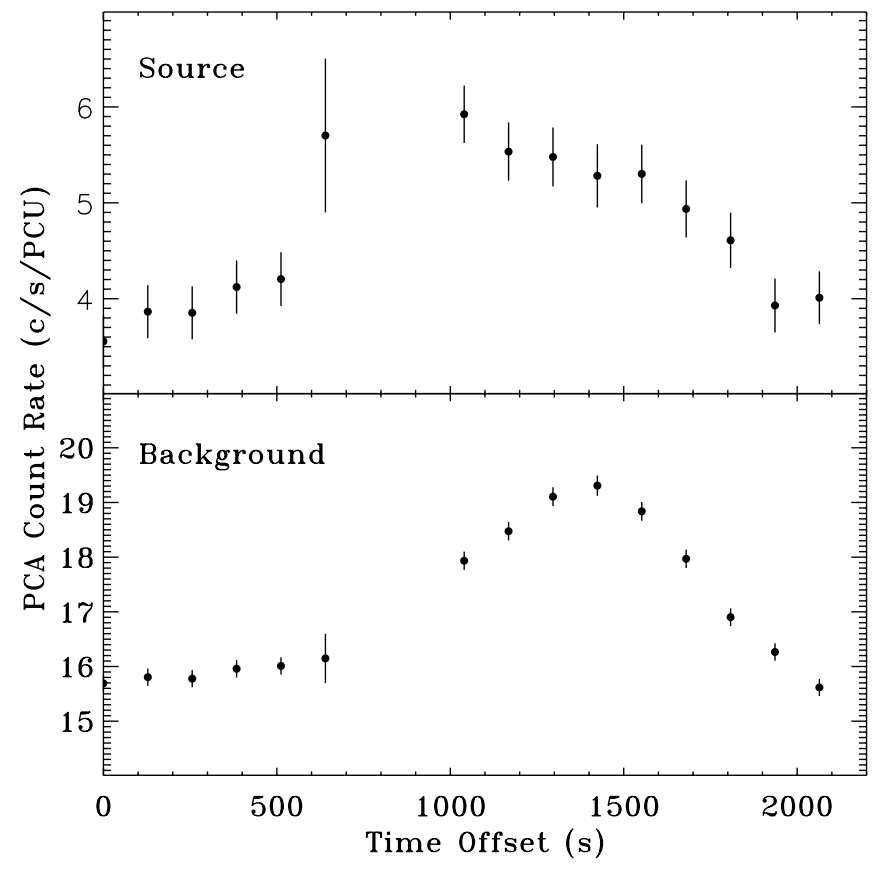

Fig. 5.-Possible rapid flare in the low state. The data were taken from one of the 1996 observations (10341-02-01-00) and was rebinned to $128 \mathrm{~s}$. The top panel shows a background-subtracted light curve of Mrk 421, while the bottom panel shows the background light curve itself for comparison. Note the similarity in the variation profiles between the two.

\subsection{Flaring State}

Mrk 421 has been quite active since 1998 (see Fig. 1). The source was regularly monitored by the RXTE during each of the major flares, with comprehensive coverage in 1998 and 2001. In general, the flaring activity also becomes more intense on short timescales (less than a day) during these periods, and quite remarkably, rapid flares (of duration less than about an hour) are often seen. Figure 6 displays a montage of light curves made from a stretch of consecutive observations during the 2001 episode. The results are quite typical of the source in the flaring state. The figure shows an impressive array of variation patterns on a wide range of timescales. Of particular interest are prominent flares that last for less than a day, which are not as obvious in the low state.

To investigate any spectral evolution during the short flares, we show in Figure 7 the corresponding hardness-intensity diagrams. Many different patterns are observed here. In some cases, the hardness ratio increases nearly monotonically with the count rate, while in some other cases the behavior is much more complicated. For example, Figures $7 b$ and $7 c$ show instances of strong hysteresis in the spectral evolution of the source. To present a more direct view, we expanded Figure $7 c$ and indicated the time progression of the evolution in Figure 8. Overall, the source appears to evolve along clockwise loops in this case. Once again, multiple branches (or subloops) reflect the coexistence of flares on a wide range of scales. Interestingly,
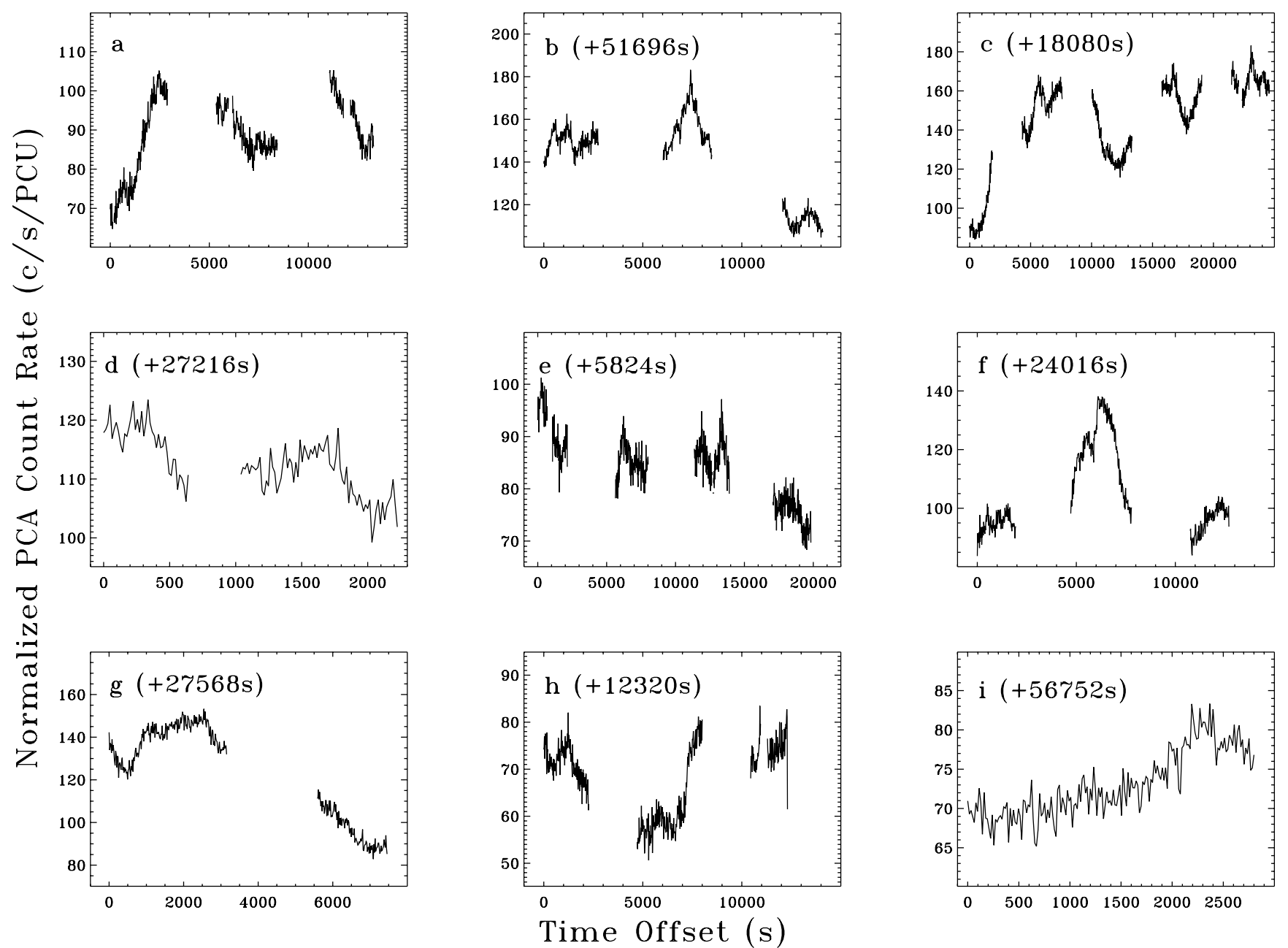

Fig. 6.- Sample light curves of Mrk 421 in the flaring state made from a stretch of consecutive observations conducted in 2001. The time is measured from the beginning of each observation; also indicated are time offsets between the starting times of adjacent observations. 

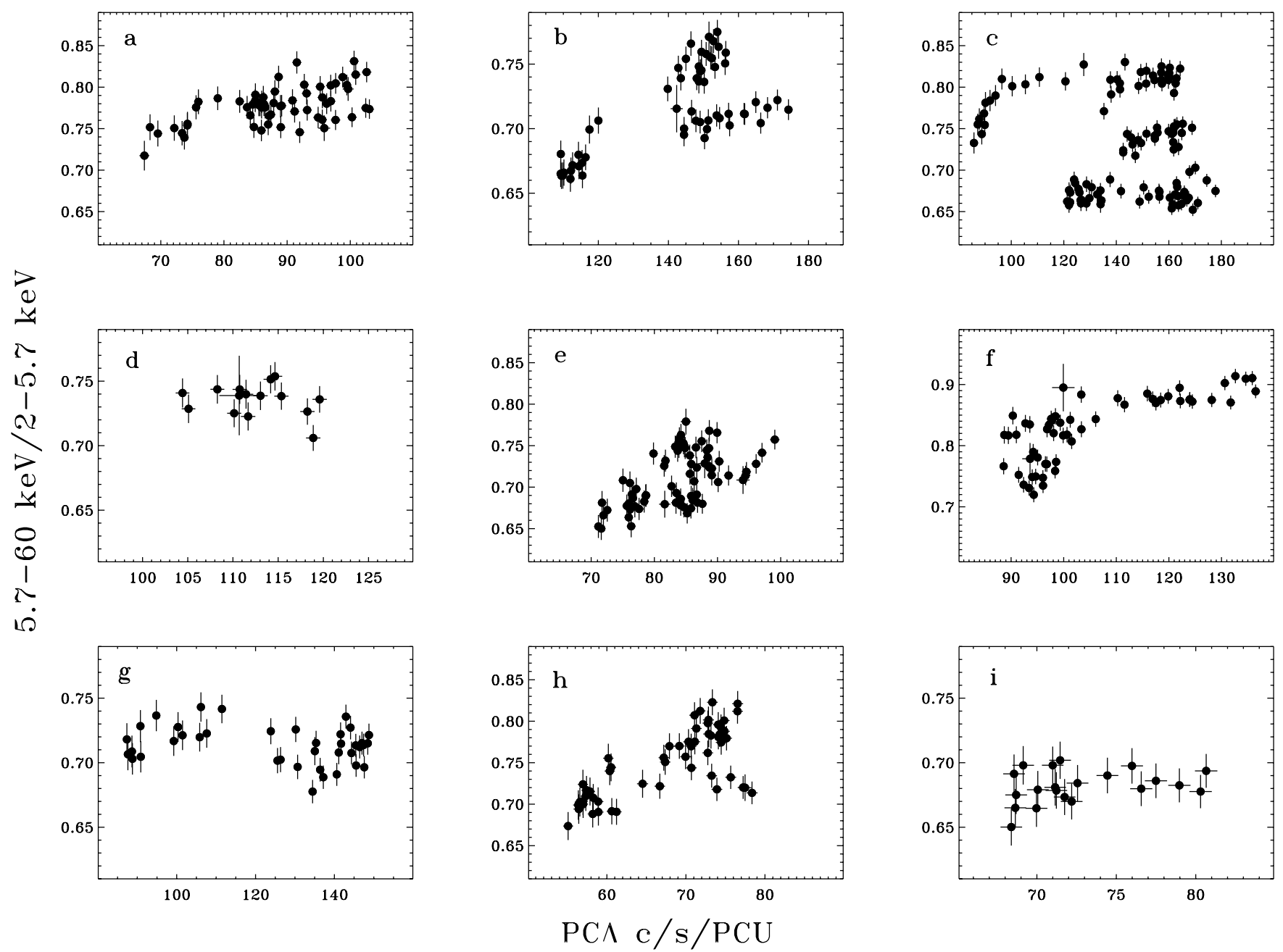

Fig. 7.- Relationship between spectral hardness and count rate. Each panel is labeled as in Fig. 6 for cross reference. The data were rebinned to $128 \mathrm{~s}$ for clarity. Note the variety of different patterns observed.

in some cases there is hardly any measurable spectral change across a flare.

The light curves in Figure 6 clearly reveal the presence of rapid flares that last for less than an hour. Figure $6 f$ shows a beautiful example of such a flare. In fact, there are significant

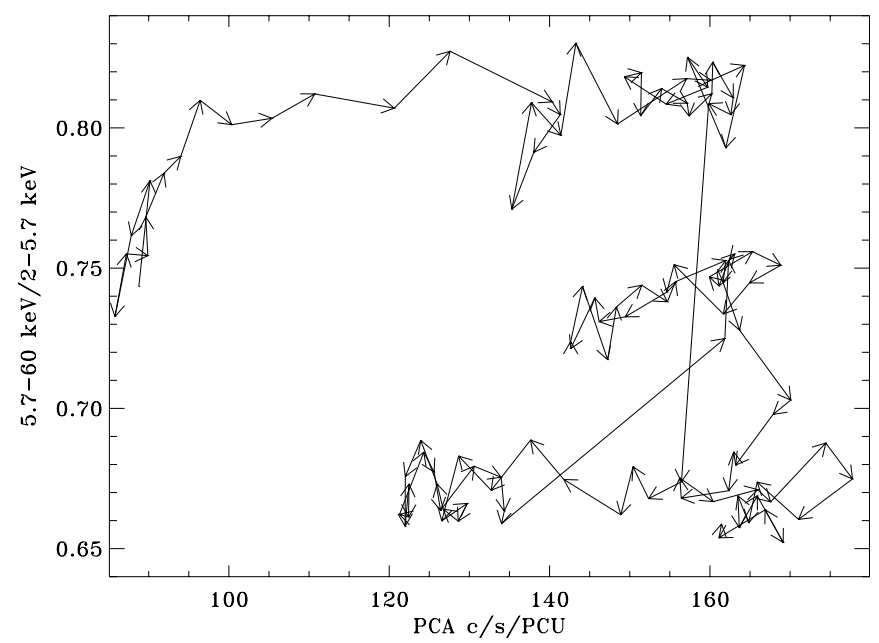

FIG. 8.-Spectral hysteresis. The figure shows an expanded view of Fig. $7 c$, but now indicates the time sequence of the evolution. substructures associated with the event, suggesting the presence of two overlapping flares of even shorter durations. Interestingly, the X-ray spectrum of the source varies little across this flare (see Fig. $7 f$ ). The effort to precisely determine the duration of each flare was, in general, complicated by the presence of data gaps, as well as by the coexistence of flares on a wide range of timescales. Sometimes, only a portion of a flare is seen. The shortest rise or decay time seen is about $1000 \mathrm{~s}$, although it can be argued that it might be even shorter in some cases (see, e.g., Fig. 6e).

\subsection{Power Density Spectra}

The observed variability may extend to shorter timescales, on which individual X-ray flares become unresolvable. The collective effects of such variability can be investigated by adopting a more sophisticated time-domain or Fourierdomain-based technique. We chose to follow the latter approach to obtain a representative power-density spectrum (PDS) of Mrk 421 in the low or flaring state. We selected a subset of the 1997 observations that are relatively long for the low state and, similarly, a subset of the 2001 observations for the flaring state. The total exposure time is comparable for the two data sets. For each observation, we made a light curve from the Standard1 data that has a time resolution of $1 / 8 \mathrm{~s}$ (but no energy resolution). We then broke the light curve into segments, 


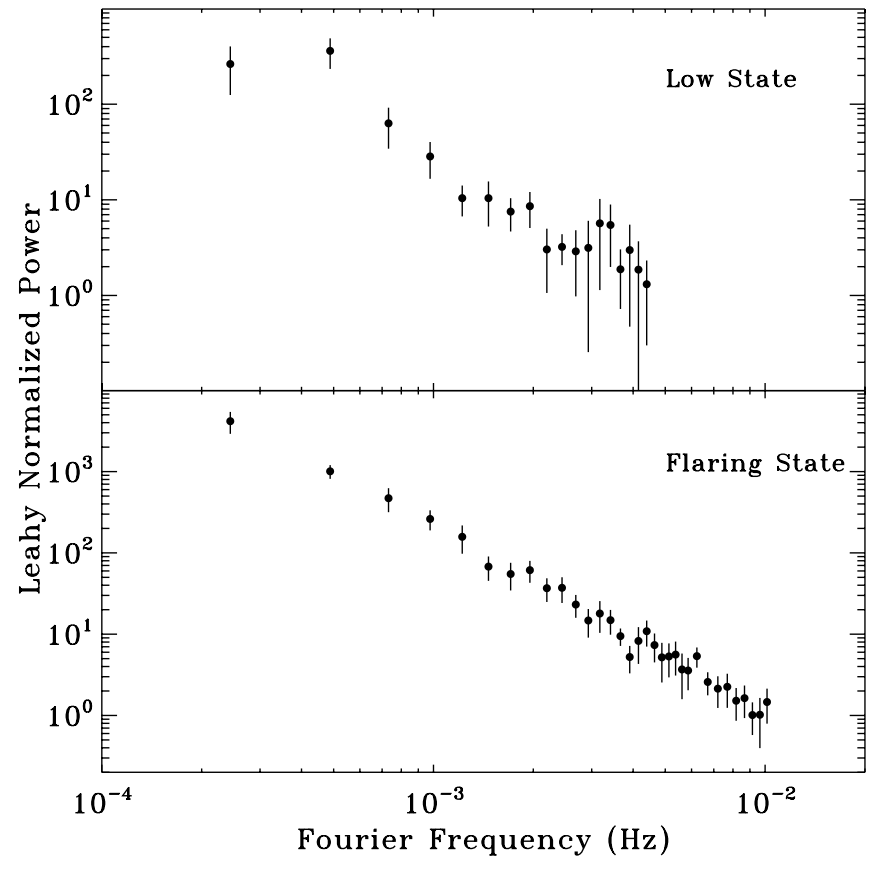

FIG. 9.-Power density spectra of Mrk 421 constructed from data with $1 / 8 \mathrm{~s}$ time resolution (see text).

each of which is $4096 \mathrm{~s}$ long (which requires the padding of data gaps or shorter segments with the average count rate). We performed a fast Fourier transformation on each segment to obtain a PDS. The PDS was normalized according to a scheme proposed by Leahy et al. (1983). The individual PDSs of the segments were then weighted (by the total number of photons) and averaged to obtain the PDS for the observation. To further improve statistics, we weighted and averaged the PDSs of the selected observations in a similar manner. From the resulted PDS, we subtracted off noise power due to Poisson counting statistics to obtain the PDS of the source. Figure 9 shows the final PDS for each state.

The observed PDS can be fitted by a simple power law, $1 / f^{\alpha}$, where $\alpha=1.9 \pm 0.2$ for the low state and $2.26 \pm 0.06$ for the flaring state, although the statistics are quite limited for the low state. The latter value is in general agreement with the published results for the flaring state (Kataoka et al. 2001; Brinkmann et al. 2003). The PDS appears to fall more steeply in the flaring state, although the difference is only of marginal statistical significance. The power-law type of PDS is typical of AGNs. What is remarkable here is that the variability of Mrk 421 is positively detected up to $0.01 \mathrm{~Hz}$ (or $100 \mathrm{~s}$ ) in the flaring state. Because of the faintness of the source, the lowstate data are much noisier. Nevertheless, the source variability is clearly seen beyond $10^{-3} \mathrm{~Hz}$.

\section{DISCUSSION}

We have observed flaring activities of Mrk 421 on a vast range of timescales (spanning roughly 4 orders of magnitude). The flares seem to occur at all times, in the low state or flaring state, and the X-ray variability seen may be entirely due to the superposition of these events on different timescales. For the first time, we have clearly resolved individual X-ray flares that last less than about an hour. They might be similar in physical origin to the TeV flare of comparable duration (Gaidos et al. 1996), albeit much smaller in amplitude. The only other blazar that is known to produce similarly rapid flares is Mrk 501 (Catanese \& Sambruna 2000).

\subsection{Multiscale X-Ray Flares}

The seemingly scale-invariant nature of flaring activities in Mrk 421 is perhaps best illustrated in Figure 10. Note the similarity in the occurrence of X-ray flares on different timescales. The detectability of individual flares on even short timescales may be limited only by the statistics of the data. Certainly, for Mrk 421, X-ray variability is still measurable on a timescale of $100 \mathrm{~s}$ (see Fig. 9). The PDS is well described by a power law (although the frequency range is limited). We note, however, that there appears to be a genuine lack of very rapid flares (with duration $<1 \mathrm{hr}$ ) in the low state. Only several candidates were identified (see Fig. 5 for an example), but their small amplitudes raise concern about possible influence by background variations.

Similar scale-invariant flaring phenomena have been observed for accreting stellar-mass black holes. For instance, the $\mathrm{X}$-ray light curves of Cyg X-1 show the presence of flares on timescales of months (e.g., Cui, Feng, \& Ertmer 2002) and "shots" on much shorter timescales (down to milliseconds; Kato, Fukue, \& Mineshige 1998 and references therein). It is often thought that the shots are associated with accretion processes in black hole candidates (BHCs), although the roles of relativistic outflows have drawn increasingly intense attention in recent years, given the ubiquitous presence of jets in such systems. It is interesting to note that a $1 \mathrm{~ms}$ flare from a $10 M_{\odot}$ black hole would roughly correspond to a $1 \mathrm{ks}$ flare from a $10^{8} M_{\odot}$ black hole, if one scales the dynamical
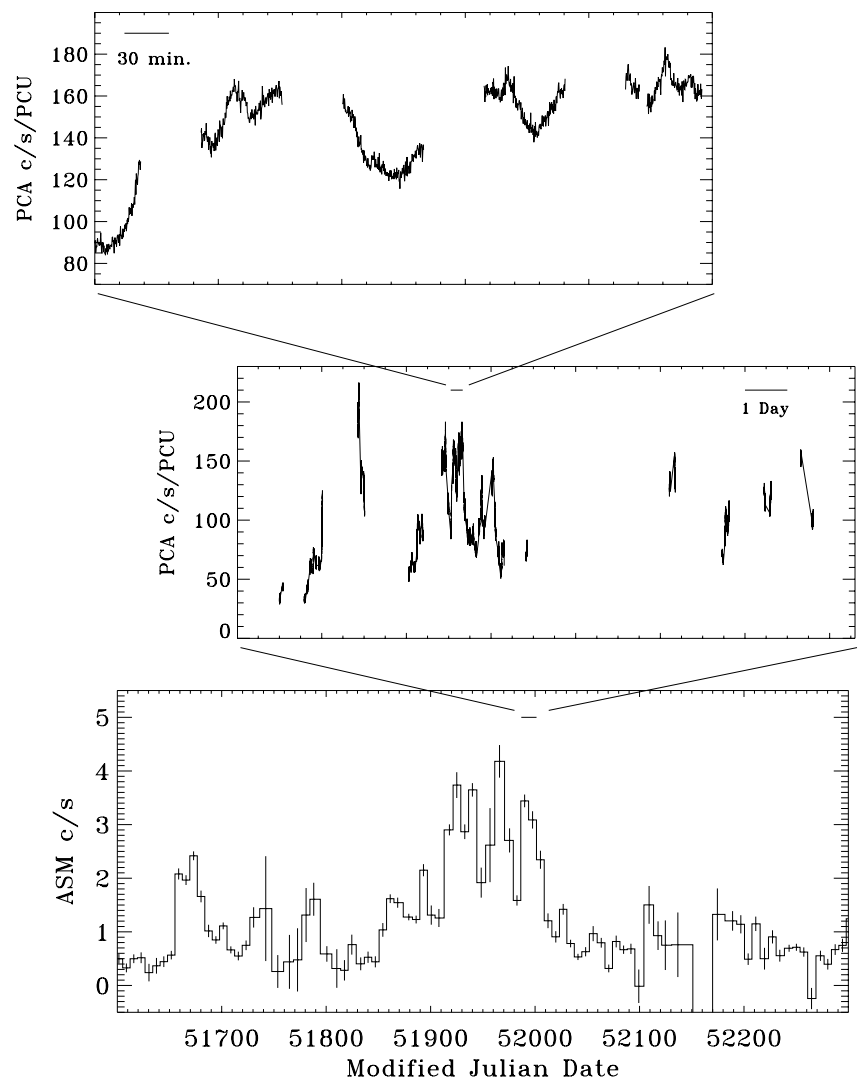

FIG. 10.-Hierarchical structure of the X-ray flaring phenomenon in Mrk 421. 
timescales by mass and takes into account the observed differences in the jet properties between BHCs (with Doppler factors $\delta \sim 1)$ and blazars $(\delta \sim 10)$. The mass of the black hole in Mrk 421 is estimated to be about $2 \times 10^{8} M_{\odot}$ (Barth, Ho, \& Sargent 2003).

Further analogies can perhaps be made between blazar flares and solar flares. To account for the power-law distribution of the solar flares, Lu \& Hamilton (1991) postulated that the coronal magnetic field in the Sun is a self-organized critical (SOC) state and that a solar flare is the result of an avalanche caused by many small magnetic reconnection events. The size of a flare is, therefore, only determined by the number of elementary reconnect events. In the SOC state, stable regions of all sizes are present in the system, so avalanches can occur on all scales (up to the size of the system), leading to the observed scale-invariant distribution of the flares. Extending the concept to BHCs, Mineshige, Takeuchi, \& Nishimori (1994) suggested that accretion disks in these systems might also be in an SOC state and that avalanches in the accretion process, triggered by, e.g., magnetic reconnection in the disk, could result in the observed distribution of shots, as well as the power-law shape of the PDS.

Lyutikov (2003) discussed the roles of magnetic reconnection in the jets of AGN and argued that magnetically dominated jets had many advantages over hydrodynamic jets, e.g., in explaining the rapid variabilities observed of blazars. Acceleration of particles may also occur in the reconnection region, and the accelerated particles then emit photons at X-ray or gamma-ray energies. It is, therefore, conceivable that the flaring activity seen in Mrk 421 might originate in the same physical process as the solar flares or flares in BHCs. Whether the magnetic field in the jets of black holes could be in an SOC state remains to be seen. Theoretical efforts to quantitatively model blazar variabilities have so far been mostly concentrated on scenarios that involve internal shocks in the jets to accelerate particles (e.g., Salvati, Spada, \& Pacini 1998; Protheroe 2002; Tanihata et al. 2003). The results are, in fact, quite encouraging. From the observational point of view, the differences between reconnection-based models and shock-based models are expected to be subtle, as discussed by Lyutikov (2003), so they will remain unresolved until better data become available. On the other hand, both types of models may also be applicable to other types of systems, including gamma-ray bursts (Lyutikov, Pariev, \& Blandford 2003) and perhaps also microquasars. Insights might be gained by looking at these physically similar systems collectively.

\subsection{Physical Constraints}

Despite the uncertainties, much can still be learned, in a relatively model-independent manner, about the physical properties of X-ray-emitting regions from the observed X-ray variability. First, all of the resolvable flares seen from Mrk 421 appear to be symmetric in shape (but see Fig. 5, if the flare is real). This implies that probably neither the synchrotron cooling time nor the particle-acceleration time is a major factor in determining the duration of a flare (Protheroe 2002). Therefore, the observed distribution of the durations of the flares may simply reflect that of the sizes of the X-ray-emitting regions, if the (jet-frame) timescale of intrinsic variability is negligible compared to the light-crossing time. The characteristic (jetframe) dimension of such a region is then given by

$$
l \approx c t_{\text {flare }} \delta /(1+z) \approx 10^{15} \mathrm{~cm} t_{\text {flare }, \mathrm{hr}} \delta_{1}
$$

where $t_{\text {flare,hr }}$ is the flare duration in units of hours, $\delta=10 \delta_{1}$, and $z$ is the redshift of Mrk $421(z=0.031)$. Given the fact that Mrk 421 is nearly always detectable at TeV energies, a large value of $\delta$ seems to always be required to sufficiently reduce the photon-photon pair-production opacity in the $\mathrm{TeV}$ emitting region (i.e., to allow $\mathrm{TeV}$ photons to escape). For instance, Gaidos et al. (1996) showed that the short duration of the observed TeV flare would imply $\delta \gtrsim 10$. Also, results from modeling the broadband SED of the source show the need for large $\delta$ (e.g., Maraschi et al. 1999; Krawczynski et al. 2001), very large in some cases ( $>50$; e.g., Krawczynski et al. 2001). However, Georganopoulos \& Kazanas (2003) argued that such extreme $\delta$ values would not be required if the jets actually decelerate on subparsec scales. In any case, equation (1) implies that the size of the emission regions probably ranges from $10^{15} \mathrm{~cm}$ to $10^{18}-10^{19} \mathrm{~cm}$. However, the equation may not hold for short-duration flares, because roughly symmetric flare profiles could also arise from situations where the synchrotron cooling time dominates and the intrinsic variability timescale is much longer than the acceleration time (Kirk \& Mastichiadis 1999). In this case, the rise or decay time of a flare would be approximately equal to the synchrotron cooling time (in the observer's frame).

Second, the observed decay time of a flare sets a firm upper limit on the synchrotron cooling time of the emitting electrons. The synchrotron cooling time is given by $\tau_{\text {syn }} \approx 6 \pi m_{e} c / \sigma_{\mathrm{T}} \gamma_{p} B^{2}$ (Rybicki \& Lightman 1979), where $m_{e}$ is the electron rest mass, $\sigma_{\mathrm{T}}$ is the Thomson cross section, $B$ is the magnetic field in the region, and $\gamma_{p}$ is the characteristic Lorentz factor of those electrons that contribute to the bulk of the observed X-ray emission (at $E_{p} \sim 10 \mathrm{keV}$, where the synchrotron peak of the SED lies). Transformed to the observer's frame, this time needs to be less than the observed decay time of an X-ray flare, i.e., $\tau_{\text {syn }} / \delta<t_{d}$, where $t_{d}$ is the decay time. This requirement leads to a lower limit on the magnetic field,

$$
B>0.88 \mathrm{G} t_{d, 3}^{-1 / 2} \delta_{1}^{-1 / 2} \gamma_{p, 5}^{-1 / 2}
$$

where $t_{d}=10^{3} t_{d, 3}$ s and $\gamma_{p}=10^{5} \gamma_{p, 5}$. The observed energies of synchrotron photons are given by $E_{p}=\delta h \nu_{c} \equiv\left(3 \mathrm{eh} / 4 \pi m_{e} c\right)$ $\delta \gamma_{p}^{2} B$ (Rybicki \& Lightman 1979), so we have $B \approx$ $5.8 E_{p, 1} \delta_{1}^{-1} \gamma_{p, 5}^{-2} \mathrm{G}$, where $E_{p}=10 E_{p, 1} \mathrm{keV}$. Substituting $B$ in equation 2, we derived an upper limit on the Doppler factor,

$$
\delta<16 t_{d, 3} E_{p, 1}^{2}\left(\gamma_{p, 5} / 3\right)^{-3}
$$

It is worth noting that $\gamma_{p}$, derived from the synchrotron selfCompton description of the observed broadband SED, is typically a few $\times 10^{5}$ (e.g., Maraschi et al. 1999; Krawczynski et al. 2001).

\subsection{Spectral Hysteresis}

We observed hysteresis associated with spectral evolution across a flare in some cases, but not all. For instance, the spectrum of Mrk 421 varied little across one of the rapid flares (see Fig. 6f). In comparison, Mrk 501 showed significant spectral variation during rapid flares (Catanese \& Sambruna 2000). In that case, the hardness-intensity diagram shows that the source followed a counterclockwise hysteresis loop (although details are more complicated).

Theoretical interpretation of the observed spectral hysteresis is not entirely clear at present. Kirk \& Mastichiadis (1999) showed, using a simplified internal shock model, that the 
hysteresis could be caused by the interplay of three characteristic times associated with synchrotron cooling $\left(\tau_{\text {syn }}\right)$, particle acceleration $\left(\tau_{\text {acc }}\right)$, and intrinsic variability $\left(\tau_{\text {var }}\right)$, respectively. Two of the four cases that they discussed might be most relevant to what we have observed in this work: (1) $\tau_{\text {var }} \gg \tau_{\text {syn }} \gg \tau_{\text {acc }}$, and (2) $\tau_{\text {var }} \approx \tau_{\text {syn }} \approx \tau_{\text {acc }}$. In the former scenario, the shape of the spectrum does not change and the intensity varies on the timescale $\tau_{\text {var }}$ (which determines the flare duration $t_{\text {flare }}$ ). This would explain the lack of spectral evolution seen during some of the flares. In the latter scenario, spectral evolution is expected. Moreover, the evolution should follow a counterclockwise hysteresis pattern in the hardness-intensity representation, since changes propagate from low energies to high energies (Kirk \& Mastichiadis 1999). Such patterns were also observed (see Fig. 4). On the other hand, the pattern shown in Figure 8 is mostly clockwise, which would require $\tau_{\text {syn }} \gg$ $\tau_{\text {var }} \gg \tau_{\text {acc }}$ or $\tau_{\text {syn }} \gg \tau_{\text {acc }} \gg \tau_{\text {var }}$ (Kirk \& Mastichiadis 1999; also see Dermer 1998). The latter seems quite unlikely, given the roughly symmetric profiles observed of the flares.

However, Li \& Kusunose (2000) pointed out that it would be difficult to draw any firm conclusions based on the sense of a hysteresis loop, because it is quite sensitive to various parameters in the model, such as the overall injection energy. By treating the Comptonization process in a consistent manner, they further showed that a hysteresis loop may change its sense going from the synchrotron-dominated regime (at low energies) to the Compton-dominated regime (at high energies). This was confirmed by Böttcher \& Chiang (2002). This is troublesome from the observational point of view, because it implies that the choice of energy bands (which is usually done without a priori knowledge about the overall SED) may affect what we see observationally. To further complicate the situation, it is nearly impossible, observationally, to cleanly isolate a flare from the hierarchical structure.

We wish to thank Mark Ertmer and Kie Li for assistance in data reduction, Feng Yuan and Markus Böttcher for useful discussions and comments on the manuscript. This research has made use of data obtained through the High Energy Astrophysics Science Archive Research Center Online Service, provided by the NASA-Goddard Space Flight Center. This work was supported in part by the NASA grant NAG5-13736.
Barth, A. J., Ho, J. C., \& Sargent, W. L. W. 2003, ApJ, 583, 134

Böttcher, M., \& Chiang, J. 2002, ApJ, 581, 127

Böttcher, M., et al. 1997, A\&A, 324, 395

Bradt, H. V., Rothschild, R. E., \& Swank, J. H. 1993, A\&AS, 97, 355

Brinkmann, W., Papadakis, I. E., den Herder, J. W. A., \& Haberl, F. 2003, A\&A, 402, 929

Buckley, J. H., et al. 1996, ApJ, 472, L9

Catanese, M., \& Sambruna, R. M. 2000, ApJ, 534, L39

Celotti, A., Fabian, A. C., \& Rees, M. J. 1998, MNRAS, 293, 239

Cui, W., Feng, Y. X., \& Ertmer, M. 2002, ApJ, 564, L77

Dermer, C. D. 1998, ApJ, 501, L157

Falcone, A. D., Cui, W., \& Finley, J. P. 2004, ApJ, 601, 165

Fossati, G., et al. 2000, ApJ, 541, 166

Gaidos, J., et al. 1996, Nature, 383, 319

Georganopoulos, M., \& Kazanas, D. 2003, ApJ, 594, L27

Giebels, B., et al. 2002, ApJ, 571, 763

Kataoka, J., et al. 2000, ApJ, 528, 243 2001, ApJ, 560, 659

Kirk, J. G, \& Mastichiadis, A. 1999, Astropart. Phys., 11, 45 Krawczynski, H., et al. 2001, ApJ, 559, 187

Leahy, D. A., et al. 1983, ApJ, 266, 160

Li, H., \& Kusunose, M. 2000, ApJ, 536, 729

\section{REFERENCES}

Lu, E. T., \& Hamilton, R. J. 1991, ApJ, 380, L89

Lyutikov, M. 2003, NewA Rev., 47, 513

Lyutikov, M., Pariev, V. I., \& Blandford, R. D. 2003, ApJ, 597, 998

Maraschi, L., et al. 1999, ApJ, 526, L81

Mastichiadis, A., \& Kirk, J. G. 1997, A\&A, 320, 19

Mineshige, S., Takeuchi, M., \& Nishimori, H. 1994, ApJ, 435, L125

Kato, S., Fukue, J., \& Mineshige, S. 1998, Black-Hole Accretion Disks (Kyoto University Press)

Protheroe, R. J. 2002, Pub. Astron. Soc. Australia, 19, 486

Punch, M., et al. 1992, Nature, 358, 477

Rees, M. J. 1978, MNRAS, 184, 61

Rybicki, G. B., \& Lightman, A. P. 1979, Radiative Processes in Astrophysics (New York: Wiley)

Salvati, M., Spada, M., \& Pacini, F. 1998, 495, L19

Spada, M., et al. 2001, MNRAS, 325, 1559

Takahashi, T., et al. 1996, ApJ, 470, L89

Tanihata, C., Takahashi, T., Kataoka, J., \& Madejski, G. M. 2003, ApJ, 584, 153

Tanihata, C., et al. 2001, ApJ, 563, 569

Urry, C. M., \& Padovani, P. 1995, PASP, 107, 803

Zhang, Y. H. 2002, MNRAS, 337, 609 